\title{
AC power measurements of electric drives: a method incorporating calorimetry and DC measurements only
}

\author{
D. Lindenthaler, G. Brasseur OVE
}

\begin{abstract}
Power analyzer specify their power measurement accuracy typically for sinusoidal signals only. In inverter applications, however, signals result from a switching operation and are therefore broadband. This article presents a method for providing a reference result for AC power measurements between inverter and motor. The reference power is determined by a combination of DC measurements and calorimetry and therefore it is independent of the AC signal's waveform.
\end{abstract}

Keywords: power measurement; electric drive; calorimeter; uncertainty evaluation

\section{AC-Wirkleistungsmessung am umrichtergespeisten Antrieb mittels Kalorimetrie und DC-Leistungsmessung.}

Aktuelle Leistungsmessgeräte für elektrische Antriebe sind typischerweise für sinusförmige Größen spezifiziert. Reale Strom- und Spannungssignale eines Inverters sind jedoch breitbandig. In dieser Arbeit wird eine Methode vorgestellt, mit welcher ein Referenzergebnis für die Wirkleistung zwischen Inverter und Motor bestimmt werden kann. Die Methode beruht auf einer Kombination aus einer DC-Leistungsmessung mit einer kalorimetrischen Verlustleistungsmessung, womit das Referenzergebnis unabhängig von der AC-Signalform ist.

Schlüsse/wörter: Leistungsmessung; elektrischer Antrieb; Kalorimeter; Messunsicherheit

Received November 11, 2016, accepted February 8, 2017, published online March 7, 2017

(c) The Author(s) 2017. This article is published with open access at Springerlink.com

\section{Introduction}

Electric drives are widespread in industry and play an increasingly important role in mobility. In order to save energy and reduce costs it is reasonable to make electric drives as efficient as possible, as they represent a significant portion of energy consumers. By optimizing the efficiency of an electric drive, measurements are required for verifying and evaluating the efficiency. A device's efficiency however, cannot be measured directly. It is determined by the ratio of the output power divided by the input power

Modern electric drives are high efficient devices with efficiencies in the range of around $90 \%$. This high efficiency causes the magnitude of in- and output power being in the same range. The efficiency determination of such devices is a challenging task as measurement uncertainties have to be considered. If in- and output power are within the same order of magnitude, power measurement uncertainties have a high impact on the computed efficiency and can lead to implausible results. The efficiency determination requires power measurements with small uncertainties, as the tolerable uncertainty of a power measurement decreases with the device's efficiency [7].

Depending on the investigated component of the drive, power has to be measured at different locations like the DC input of the inverter, the AC power between motor and inverter or the mechanical motor power. This article addresses power measurements between inverter and motor at automotive drivetrains, denoted by $P_{a c}$ in Fig. 1. This AC power is used for evaluating the efficiency of an inverter or motor, serving as input- or output power depending on the measurement task.

For measuring power in inverter-fed drives, power analyzer are typically utilized. A power analyzer samples and multiplies voltage and current and averages the instantaneous power for computing the active power. Besides measurement issues like the accuracy of a probe, this sample based approach yields to two additional measurement problems: the bandwidth of the measurement equipment has to include the signal bandwidth and a suitable averaging interval has to be chosen to average the instantaneous power.

Choosing a suitable averaging interval is especially challenging for dynamic loads like acceleration in an automotive drive. Generally, there are multiple factors influencing the voltage and current signals on the $A C$ side: switching frequency of the inverter, rotational speed of the motor, nonlinearities of the control algorithm and dynamics of the load. As a result of the multiple influencing factors, it is possible that the overall resulting period of the AC voltage and current signals can be unexpectedly long and multiple times larger than the mechanic cycle time of the motor. In this case, the correct averaging interval has to be larger as hundred times the rotor period. Measuring power with dynamic loads however is not the scope of the current article; this article addresses static load points only. If the motor load is static, then the integration interval issue can be solved by choosing the integration interval suitable large.

The second measurement issue is the non-sinusoidal shape of voltage and current on the AC side of the inverter. These signals are directly affected by the switching operation of the inverter and

Lindenthaler, David, Institute of Electrical Measurement and Measurement Signa Processing, Graz University of Technology, Inffeldgasse 23/II, 8010 Graz, Austria (E-mail: lindenthaler@tugraz.at); Brasseur, Georg, Institute of Electrical Measurement and Measurement Signal Processing, Graz University of Technology, Inffeldgasse 23/II, 8010 Graz, Austria (E-mail: georg.brasseur@tugraz.at) 


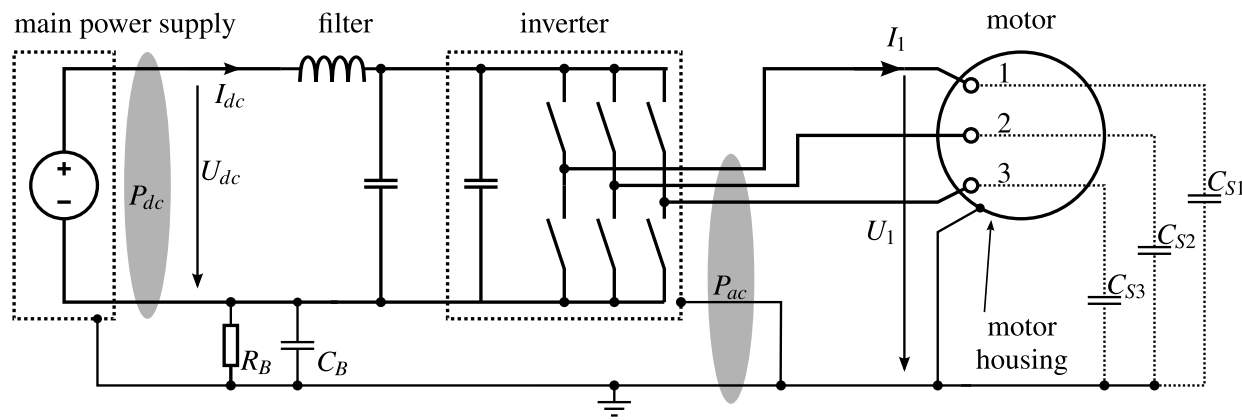

Fig. 1. Electric drive with a three phase inverter and a motor in an automotive application. All housings are bonded to ground via chassis. The negative power supply output is connected to ground for safety reasons with a high ohmic bonding resistor $R_{B}=370 \mathrm{k} \Omega$ and a bonding capacitor $C_{B}=3 \mu \mathrm{F}$. The capacities $C_{S 1}, C_{S 2}$ and $C_{S 1}$ represent the stray capacitance of cabling, inverter and motor winding for each motor phase. The LC filter stage minimizes AC content of the current $I_{d c}$ and the voltage $U_{d c}$

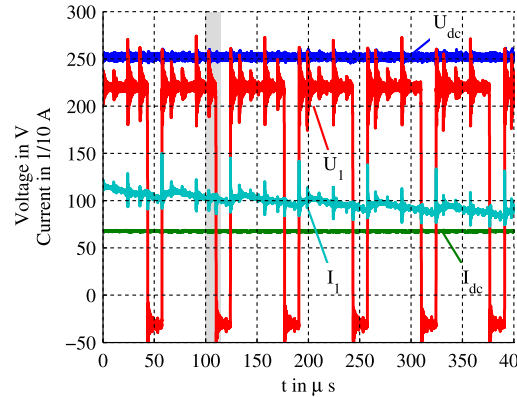

(a) Switching pattern

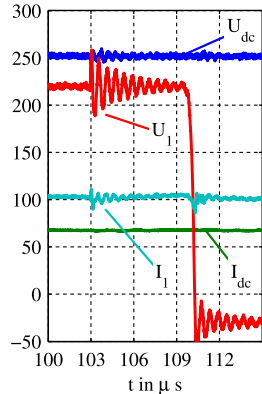

(b) Zoom
Fig. 2. Measured voltage and current signals on the $A C$ and $D C$ side of the inverter with a sampling frequency of $125 \mathrm{MHz}$. The AC voltage and current contain overshoots resulting from the switching operation of the inverter. All measurement probes are equipped with common mode chokes, reducing noise created by inverter switching. The offset of the $A C$ voltage $U_{1}$ of $-30 \mathrm{~V}$ results from the capacitive voltage divider with the capacitor $C_{B}$ and the sum of all stray capacities $C_{S}$

therefore have a wide bandwidth. Figure 2 illustrates exemplary voltage and current signals of the utilized drive. The AC voltage signal $U_{1}$ in Fig. 2 results from the switching operation of the inverter and is approximately rectangularly shaped while the $A C$ current $/ 1$ is roughly piecewise linear. Both, AC voltage and current, include strong overshoots extending the signal bandwidth as can be seen in the zoom of Fig. 2b. These overshoots result from the switching of the inverter, which excites resonant circuits composed by the setup of motor, inverter and cabling $[1,6]$ and represented by the stray capacities $C_{S 1}, C_{S 2}, C_{S 3}$ and the motor and cable inductances. The resonant frequency can range from some $100 \mathrm{kHz}$ up to some $10 \mathrm{MHz}$ depending on the setup. A power analyzer should accurately capture this high frequency signal components for correctly determining the active power.

Because of the non-sinusoidal waveforms a further issue arises with power measurements on the AC side: power analyzer specify their uncertainty basically as a function of amplitude, frequency and power factor of pure sine waves. One major drawback of this approach is that neither the $A C$ current nor the $A C$ voltage are pure sine waves in an inverter application. Both signals result from the switching operation and are composed by multiple spectral components. For an uncertainty evaluation it is not defined which fre- quency has to be considered for computing the power analyzer's uncertainty. The signal component with the highest spectral contribution is the fundamental for the current and the inverter's switching frequency for the voltage. In this article, the measurement uncertainty is therefore computed by considering the switching frequency and the fundamental current.

Current literature on digital sampling wattmeter pays particular attention to a high measurement bandwidth. In the article [4] for example, a metrology device is characterized for frequencies up to $1 \mathrm{MHz}$. Commercially available devices provide a larger bandwidth ranging from $1 \mathrm{MHz}$ to $10 \mathrm{MHz}$ in exchange for a lower accuracy. However, power measurements with these devices are specified for sinewaves or a limited combination of sinewaves. A calorimetric approach exists for power measurements at electric drives besides the digital sampling approach $[2,8]$. The calorimetric method measures directly heat losses. But calorimeters are either bulky or suffer from accuracy if large AC output power from an inverter is measured directly.

The current article proposes a novel method for providing a reference power result for the AC power to overcome the drawback of the power analyzer's limited bandwidth as well as the limited power measurement range of the calorimetric approach. The reference result for the AC power is determined by combining a DC power measurement with a calorimetric heat loss measurement. As the proposed method does not require to measure AC quantities, the reference result provides to two important properties: is neither affected by a bandwidth limitation error nor by an error due to a wrong averaging interval. This desirable properties are achieved as the proposed method provides inherently DC quantities. Therefore, the obtained AC power measurement will serve as reference result for further investigations on power analyzers' accuracy even for dynamic load changes.

This article is structured as follows: Sect. 2 describes the test stand with the calorimetric measurement setup and derives the measurement uncertainty. Measurement scenarios and results are discussed in Sect. 3 and the main findings are summarized in Sect. 4.

\section{Calorimetric $A C$ power measurements}

This section describes three aspects of the setup: the electric drive, the calorimetric measurement unit and the uncertainty evaluation of the calorimetric AC power measurements.

2.1 Electric drive and power measurement test stand The components of the drive are: a DC power source, a three phase inverter driving a permanent magnet synchronous motor (PMSM) 


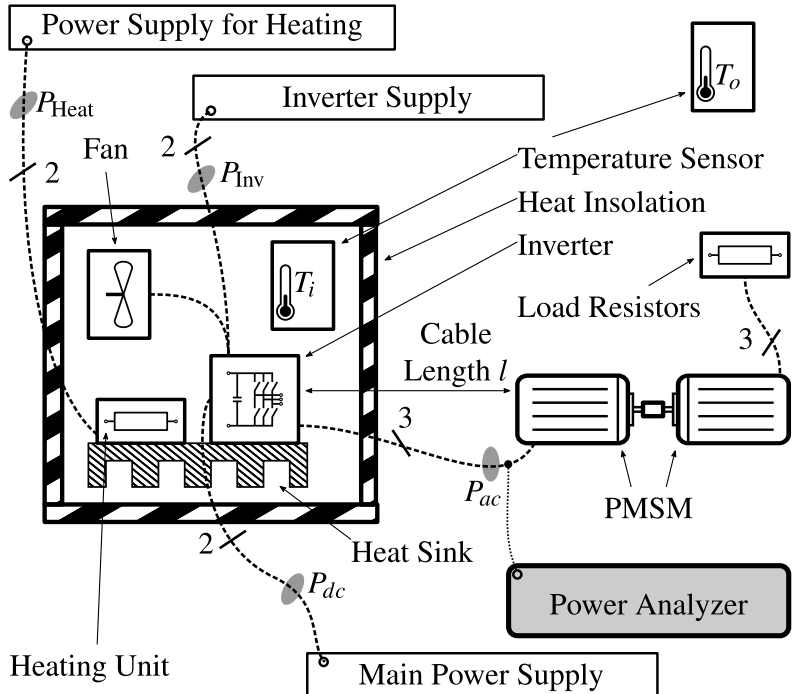

Fig. 3. Components for calorimetric AC power measurements. The complete setup is placed in a climate chamber for having constant ambient temperature $T_{0}$. In the first stage, the inverter drives the motor and rises the interior temperature of the box. In the second stage, the inverter is in standby and the heating unit is controlled such that the same temperature rise prevails

connected via a jaw-type coupling with a second PMSM acting as generator. The generator is connected to load resistors converting the generator's torque into heat energy. These components are wired according to Fig. 3 and Fig. 1.

The negative power supply output is connected to ground via a high ohmic resistor $R_{B}$ and a capacitor $C_{B}$ as shown in Fig. 1. By doing so, the test stand grounding scheme complies with automotive safety guidelines.

The main power supply is realized by a DC power source providing an isolated $D C$ output voltage $U_{d c}$ directly wired to the inverter. An individual microcontroller board based on an ARM Cortex-M4 chip provides the control signals for the inverter. The microcontroller implements a field oriented control algorithm for the PMSM with current and speed control. The underlying switching pattern of the field oriented control algorithm is a space-vector modulation scheme with two equal length zero states per cycle.

\subsection{Calorimete}

The working principle is based on the fact that the rise of temperature in an $\mathrm{AC}$ load relies exclusively on active power. To determine the power, this temperature rise is compared to the temperature rise resulting from a $D C$ power source. If the rate of temperature change is equal for the $A C$ and $D C$ case, both power losses have to be identical and independent of the spectral composition of voltage and current signals as long as their common signal period is much smaller than the time constant of the calorimeter. Further details on the different variants of calorimeters, their properties and a categorization is given in [2].

Based on the classification in [2], a closed, balanced type calorimeter is utilized to determine the electric power on the $A C$ side of the inverter. The realized calorimeter is illustrated in Fig. 3 and consists of a wooden box serving as thermal insulator, two temperature sensors for determining the temperature difference between in- and outside the wooden box, an electric heating unit and a fan inside the box securing a homogeneous temperature distribution. The picked temperature sensors provide an absolute accuracy of $\pm 0.5^{\circ} \mathrm{C}$. For balanced type calorimeters however a small hysteresis error is more important which is $\pm 2 \cdot 10^{-3}{ }^{\circ} \mathrm{C}$ for the picked sensors.

The calorimeter determines the power on the AC side of the inverter $P_{C a c}$ in two stages: in the first stage the drive is in normal operation mode and the temperature inside the box $T_{i}$ rises due to heat loss. The temperature rise results from the sum of all electric power entering the insulated box, the thermal inertia of the components in the box and the heat transfer rate to the outside of the box denoted by $R_{\mathrm{Th}}\left(\left[R_{\mathrm{Th}}\right]=\mathrm{K} M\right)$. After an initial transient time, the temperature difference $\delta_{1}$ between the inside temperature of the box $T_{i}$ and outside temperature of the box $T_{0}$ is steady. The transient time depends on the time constant of the calorimeter. For the constructed calorimeter, the time constant to obtain $63 \%$ of the final value is approximately 30 min resulting in a transient time of $3 \mathrm{~h}$. The steady state temperature difference $\delta_{1}$ is:

$$
\delta_{1}=\left.\left(T_{i}-T_{0}\right)\right|_{\text {motor running }}=R_{\mathrm{Th}} \cdot\left(P_{d c}-P_{\mathrm{Cac}}+P_{\mathrm{Inv} 1}\right) .
$$

In Eq. (1), $P_{\text {Inv1 }}$ denotes the auxiliary power of the inverter. The AC power $P_{C a c}$ is subtracted is subtracted from the DC power $P_{d c}$ as it channels through the thermally insulated box. After the initial transient temperature rise, the drive is deactivated and the temperature difference $\delta_{1}$ is saved for the next measurement stage.

The second stage of the measurement process is the heating stage, which follows immediately the first stage. In this stage, the heating unit inside the box is powered by a temperature controlled DC source such that the temperature difference $\delta_{2}$ prevails the same temperature difference $\delta_{1}$ as during the first running-motor stage:

$$
\delta_{2}=\left.\left(T_{i}-T_{0}\right)\right|_{\text {heating }}=R_{\text {Th }} \cdot\left(P_{\text {Heat }}+P_{\text {Inv2 }}\right)
$$

The AC power $P_{C a c}$ results from subtracting Eq. (1) from Eq. (2):

$$
P_{\mathrm{Cac}}=P_{\mathrm{dc}}+P_{\mathrm{Inv} 1}-P_{\text {Heat }}-P_{\mathrm{lnv} 2}-\frac{\delta_{1}-\delta_{2}}{R_{\mathrm{Th}}} .
$$

Under ideal conditions the last term ${ }^{\delta_{1}-\delta_{2} / R_{\mathrm{Th}}}$ will cancel out; in reality however, the magnitude of ${ }^{\delta_{1}-\delta_{2}} / R_{\mathrm{Th}}$ is at least very small. Hence, it is sufficient to measure $D C$ power and temperatures for determining the active $A C$ power on the $A C$ side of the inverter (see Eq. (3)). For precise measurements, it is important that the ambient conditions and the setup of the calorimeter do not change between the two stages. Therefore, the complete setup is housed in a climate chamber with a constant temperature $T_{0}$ of $18^{\circ} \mathrm{C}$ and the wooden box and its cabling is not modified between the two measurement stages.

The measurement of the inverter's DC input power $P_{d c}$ is realized by means of measuring the $D C$ voltage $U_{d c}$ and the voltage drop $U_{S d c}$ across a high precision current shunt. Two Keithley 2100 6.5 digit voltmeters are used. As the voltmeters are specified for DC signals, no AC ripple is allowed. Therefore, the DC link capacitor of the utilized inverter is equipped with an additional LC filter stage. This filter stage minimizes $A C$ content of the voltage and current signals.

\subsection{Error analysis}

The objective of the article is to provide a reference result for the AC power, which is free of the power analyzer's bandwidth limitation error and errors resulting from a wrong integration interval. In order to compare the calorimetric $\mathrm{AC}$ power measurement with the result of the power analyzer, the upper bound for the uncertainty of 
the calorimetric $A C$ power $P_{C a c}$ is derived by error propagation

$$
\begin{aligned}
\Delta P_{\mathrm{Cac}}= & \Delta P_{d c} \\
& +\underbrace{\Delta P_{\text {Inv1 }}+\Delta P_{\text {Inv2 }}+\Delta P_{\text {Heat }}+\frac{\Delta \delta}{R_{\text {Th }}}}_{\Delta P_{\text {Cal }}}+\frac{\delta_{1}-\delta_{2}}{R_{\text {Th }}^{2}} \Delta R_{\text {Th }}
\end{aligned}
$$

In Eq. (4), the uncertainty can be split into two parts. The first part is the uncertainty of the $\mathrm{DC}$ power measurement $\Delta P_{d c}$ incorporating the two voltmeter uncertainties and the $D C$ current shunt uncertainty. The second part $\Delta P_{\mathrm{Cal}}$ results from the calorimetric measurement. The uncertainty of the inverter supply power during the two measurement stages $\Delta P_{\text {Inv1 } 1}$ and $\Delta P_{\text {Inv2 }}$ follows from the individual measurement hardware on the microcontroller board and $\Delta \delta$ corresponds to a non-ideal steady-state temperature during the heating stage and hysteresis errors of the temperature sensors. Uncertainties of the thermal resistance $R_{\text {Th }}$ are not considered, as the influence of $\Delta R_{\mathrm{Th}}$ on $P_{\mathrm{Cac}}$ is almost negligible. Additionally, the thermal resistance $R_{\mathrm{Th}}$ is constant during one measurement because of the balanced calorimetric measurement principle.

The uncertainty bounds of the power analyzer's result $\Delta P_{a c}$ are usually a function of the effective voltage and current, the frequency range of the signals and the power factor. As described in Sect. 1, the power analyzer's uncertainty is determined from the power analyzer's user manual by considering the inverter's switching frequency for the voltage and the fundamental current frequency for the current.

\section{Experiments}

Turning now to the experimental section of this article, in which the AC power of the calorimetric method is compared with the power analyzers result.

\subsection{Investigated experiments}

The experiments include a parameter variation of the brake motor's load and the activation of the power analyzer's anti-aliasing filter (AAF). For all experiments the main power supply DC voltage $U_{d c}$ is constant at $250 \mathrm{~V}$ and the motor speed control is set to a mechanical speed of $1050 \mathrm{rpm}$, resulting in a fundamental frequency of the motor current of $70 \mathrm{~Hz}$ (4 pole pairs). The assembly of the test stand results in a cable length of $1.25 \mathrm{~m}$ between inverter and motor and the inverter uses a switching frequency of $15 \mathrm{kHz}$.

\subsection{Utilized power analyzer}

The power analyzer utilized is a Fluke Norma 5000 with power phase channels of type PP50. The sampling frequency of the device is $1.024 \mathrm{MHz}$ with a bandwidth of $10 \mathrm{MHz}$ and the activatable AAFs have a cutoff frequency of $100 \mathrm{kHz}$. The last calibration of the power analyzer was performed in 2012. Because of the out-of-date calibration, all following results should be taken on a relative scale, such that the results are comparable among each other rather than absolutely.

According to the power analyzer's reference manual [3, sect. 11], the $A C$ uncertainty for voltage and current signals is specified as a function of the signal rms range ( $r n g$ ), the rms reading ( $r d g$ ) and the signal frequency. In this article, the frequency of the spectral component with the largest amplitude is used for determining the uncertainty. The relative current uncertainty $F_{i}$ in the frequency range from $10 \mathrm{~Hz}$ to $1000 \mathrm{~Hz}$ has an uncertainty of $\pm(0.05 \%+$ $0.05 \% \mathrm{rng} / \mathrm{rdg}$ ). In contrast to the current signal, most signal power of the voltage signal is located at the spectral component of the inverter's switching frequency. Therefore the relative voltage
Table 1. Experiments for evaluating the AC power measurements

\begin{tabular}{|c|c|c|c|c|c|}
\hline \multirow[t]{2}{*}{ Nr. } & \multirow[t]{2}{*}{ AAF } & \multicolumn{3}{|l|}{ Calorimetry } & \multirow{2}{*}{$\begin{array}{l}\text { Power analyzer } \\
P_{a c} \pm \Delta P_{a c} \\
(W)\end{array}$} \\
\hline & & $\begin{array}{l}\overline{P_{C a c} \pm \Delta P_{C a c}} \\
(\mathrm{~W})\end{array}$ & $\begin{array}{l}\Delta P_{d c} \\
(W)\end{array}$ & $\begin{array}{l}\Delta P_{\mathrm{Cal}} \\
(\mathrm{W})\end{array}$ & \\
\hline 1 & off & $1584.98 \pm 2.18$ & 1.39 & 0.79 & $1583.86 \pm 12.29$ \\
\hline 2 & on & $1584.77 \pm 2.18$ & 1.39 & 0.79 & $1581.43 \pm 12.4$ \\
\hline 3 & off & $483.43 \pm 1.25$ & 0.46 & 0.79 & $7 \pm 3.74$ \\
\hline 4 & on & $483.33 \pm 1.25$ & 0.46 & 0.79 & $480.45 \pm 3.76$ \\
\hline
\end{tabular}
with the power analyzer and the calorimetric measurement

measurement uncertainty $F_{V}$ is determined for a frequency of $15 \mathrm{kHz}$ and it is $\pm(0.235 \%+0.235 \% \mathrm{rng} / \mathrm{rdg})$.

The uncertainty per motor phase $\Delta P_{n}$ is computed according to the formula given in the power analyzer's reference manual $[3, p$. 11-5]. The reference manual's formula sums the relative voltage and current uncertainty and a further expression incorporating phase uncertainty $k_{a}$, range to reading ratio and power factor $\lambda$ :

$$
\begin{aligned}
& \Delta P_{n}=P_{n} . \\
& \quad\left(F_{v}+F_{i}+\frac{\pi \cdot k_{a}}{180} \cdot \sqrt{\max \left(1 ; \frac{\mathrm{rng}_{v}}{\mathrm{rdg}_{v}} ; \frac{\mathrm{rng}_{i}}{\mathrm{rdg}}\right) \cdot \frac{1-\lambda^{2}}{\lambda^{2}}}\right) \\
& n \in\{1,2,3\} .
\end{aligned}
$$

In Eq. (5), $P_{n}$ denotes the power analyzer's active power per phase $n$ and the phase uncertainty $k_{a}$ depends on a signal frequency. As the largest contribution to the active power is the spectral component of the fundamental, the phase uncertainty $k_{a}$ is determined with a frequency of $70 \mathrm{~Hz}$ and equals to $k_{a}=5.35 \cdot 10^{-3 \circ}$. The maximum operator max in Eq. (5) gives the maximum value of its three inputs. The power factor $\lambda$ is for all experiments approximately constant with $\lambda=0.3$. Summing up the power measurement uncertainty $\Delta P_{n}$ of each motor phase results in the measurement uncertainty of the power analyzer $\Delta P_{a c}$ :

$$
\Delta P_{a c}=\sum_{n=1}^{3} \Delta P_{n}
$$

\subsection{Results}

The settings and results of the experiments are listed in Table 1 and illustrated in Fig. $4 \mathrm{a}$ and Fig. 4b. The two colors stand for the AC power $P_{a c}$ directly determined by the power analyzer and the AC power obtained by the calorimetric method $P_{C a c}$. For both measurement results the corresponding worst case uncertainty bound is shown. The first half of experiments shown in Fig. 4a cover a high power brake load with approximately $1.6 \mathrm{~kW}$ inverter AC power. The experiments in Fig. 4b illustrate the results of the low power load point with approximately $480 \mathrm{~W}$.

A common result of all experiments is, that the uncertainty bounds of the calorimetrically determined $A C$ power $P_{C a c}$ are smaller than those directly obtained from the power analyzer, as shown in Table 1. By comparing the high power load points from experiment number 1 and 2 with the low power experiments it turns out that the uncertainty bounds of both methods increase differently: a four times higher $A C$ power results in a four times higher uncertainty bound of the power analyzer $\Delta P_{a c}$. In contrast, the calorimetric uncertainty just increases by a factor of around two. The smaller increase can be explained by analyzing Eq. (4). The error bounds of the calorimetric AC power $P_{C a c}$ calculates 


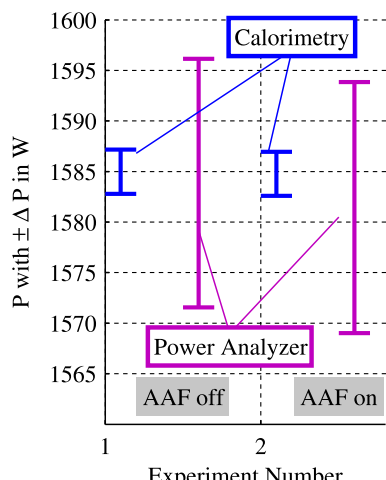

Experiment Number

(a) High power operation point.

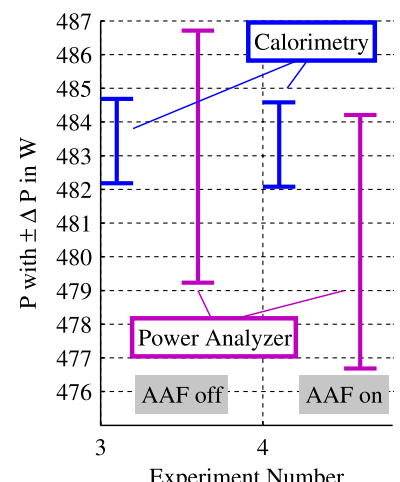

(b) Low power operation point
Fig. 4. Results for two operation points at $250 \mathrm{~V}$ DC voltage. The two colors denote the $\mathrm{AC}$ power obtained by the calorimetric method $P_{C \text { ac }}$ and the AC power directly determined by the power analyzer $P_{a c}$. (Color figure online)

from the error bounds of the DC power measurement $\Delta P_{d c}$ plus the lumped expressions $\Delta P_{C a l}$ resulting from the calorimetric measurement principle. The uncertainty of the DC power measurement $\Delta P_{d c}$ comes from the voltmeters and the current shunt inaccuracy. Depending on the DC current range, the DC power measurement uncertainty $\Delta P_{d c}$ scales with approximately $0.085 \%$. The calorimetric uncertainty $\Delta P_{\mathrm{Cal}}$ (see Eq. (4)) comes from the inverter supply measurement uncertainty $\Delta P_{\ln v 1}$ and $\Delta P_{\ln v 2}$, the uncertainty of the $D C$ heat power $\Delta P_{\text {Heat }}$ and imperfections of the temperature compensation $\frac{\Delta \delta}{R_{\mathrm{Th}}}$. The largest contribution to the uncertainty $\Delta P_{\mathrm{Cal}}$ comes from imperfections of the temperature compensation $\frac{\Delta \delta}{R_{\mathrm{T}}}$. A proportional-integral controller compensates for the temperature rise independently of the load point, resulting in an almost constant uncertainty $\Delta P_{\mathrm{Cal}}$ of $790 \mathrm{~mW}$. Therefore, the calorimetric method provides high accuracy over a wide measurement range as long as the constant error term is sufficiently small compared to the AC power

As can be seen from Fig. 4, the results from the calorimetric method and the power analyzer do overlap for all experiments. Therefore, it can be concluded, that both methods produce, within their uncertainty bound, correct results. However, a difference of $2.5 \mathrm{~W}$ in the power analyzer's result can be observed by comparing experiment number 1 with 2 . The same difference in $P_{a c}$ can be found by comparing the low power operation point in experiment number 3 with 4 . The only difference between experiment number 1 and 2 and experiment number 3 and 4 is the activation of the power analyzer's AAF. Hence, the most reasonable interpretation is, that the AAF with its $100 \mathrm{kHz}$ cutoff frequency attenuates high frequency signal components which contribute to the $A C$ power. The finding is consistent with a previous study of the authors [5], which analyzes the influence of high frequency signal components on power measurements. Both, the previous study and the current article show that a limited measurement bandwidth can result in a power measurement error in the range of some watt.

In order to evaluate the effect of activating the power analyzer's AAF in the current setup, the AC signals of the test stand are further analyzed. A spectral analysis with an oscilloscope and careful suppression of electromagnetic interference showed ringing of the AC signals. The dominant frequency of the overshoot depicted in
Fig. $2 \mathrm{~b}$ is $1.7 \mathrm{MHz}$. The power dissipated in this frequency range is only recognized by the power analyzer if the AAF is deactivated.

Basically, the power analyzer's bandwidth should not be reduced by activating the $A A F$, if accurate power measurements are required. Deactivating the power analyzers AAF allows for measuring broadband signals from an inverter with the widest bandwidth available. A deactivated AAF will result in aliasing, as the power analyzer samples with a rate of $1 \mathrm{MHz}$ and its bandwidth is $10 \mathrm{MHz}$. Therefore, it can be essential to activate the AAF for measurements like harmonic analysis of voltage and current signals for example. The results show however, that the aliasing error of the power analyzer has a smaller influence on power measurements as a bandwidth limitation error. Therefore, it is recommended to utilize the full measurement bandwidth of a power analyzer, if accurate power measurements are required.

\section{Conclusion}

The objective of this article is to present a method to determine the active $A C$ power at an electric motor. The key strength of the presented method is, that the active $A C$ power is obtained without measuring AC signals, but to measure the DC supply power reduced by the measured power losses of inverter plus auxiliaries. The power loss measurement uses a calorimetric method which is inherently independent of the signal's bandwidth and shape. As shown in the experimental section, the uncertainty bounds of the presented method are smaller than the power analyzer's bounds. Therefore, the article provides a reference result for further investigations on power measurements on the $A C$ side of an inverter.

\section{Acknowledgements}

Open access funding provided by Graz University of Technology.

Open Access This article is distributed under the terms of the Creative Commons Attribution 4.0 International License (http://creativecommons.org/ /licenses/by/4.0/), which permits unrestricted use, distribution, and reproduction in any medium, provided you give appropriate credit to the original author(s) and the source, provide a link to the Creative Commons license, and indicate if changes were made.

\section{References}

1. Boglietti, A., Cavagnino, A., Lazzari, M. (2007): Experimental high-frequency parameter identification of AC electrical motors. IEEE Trans. Ind. Appl., 43(1), 23-29. doi:10.1109/ TIA.2006.887313

2. Cao, W., Asher, G. M., Huang, X., Zhang, H., French, I., Zhang, J., Short, M. (2010): Calorimeters and techniques used for power loss measurements in electrical machines. IEEE Instrum. Meas. Mag., 13(6), 26-33. doi:10.1109/MIM.2010.5669610.

3. Fluke Corporation: NORMA 4000/5000 power analyzer (2007). Operators Manual, Rev. 2.

4. Houtzager, E., Rietveld, G., van den Brom, H. (2013): Switching sampling power meter for frequencies up to $1 \mathrm{MHz}$. IEEE Trans. Instrum. Meas., 62(6), 1423-1427. doi:10. 1109/TIM.2013.2250151.

5. Lindenthaler, D., Brasseur, G. (2015): Signal-bandwidth evaluation for power measurements in electric automotive drives. IEEE Trans. Instrum. Meas., 64(6), 1336-1343. doi:10.1109/TIM.2015.2419052.

6. Ogasawara, S., Akagi, H. (1996): Modeling and damping of high-frequency leakage currents in PWM inverter-fed ac motor drive systems. IEEE Trans. Ind. Appl., 32(5), 1105-1114. doi:10.1109/28.536872.

7. Pasini, G., Peretto, L., Tinarelli, R. (2013): Study of the accuracy requirements of the instrumentation for efficiency measurements in power conversion systems. IEEE Trans. Instrum. Meas., 62(8), 2154-2160. doi:10.1109/TIM.2013.2264864.

8. Weier, S., Shafi, M. A., McMahon, R. (2010): Precision calorimetry for the accurate measurement of losses in power electronic devices. IEEE Trans. Ind. Appl., 46(1), 278284. doi:10.1109/TIA.2009.2036667. 


\section{Authors}

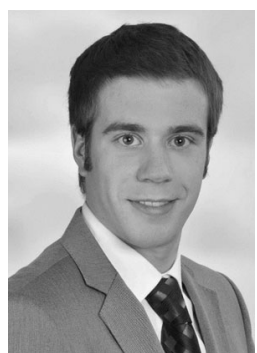

\section{David Lindenthaler}

received the B.Sc. and Dipl.-Ing. degrees in electrical engineering from Graz University of Technology (TU Graz), Austria, in 2010 and 2013, respectively. He is currently a research and teaching assistant with the Institute of Electrical Measurement and Measurement Signal Processing, TU Graz, where he is pursuing the Ph.D. degree in information and communications engineering. His research interests include measurement uncertainties, signal processing and measurement technology for electric power measurements in electric drives.

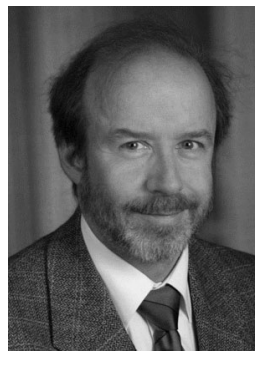

\section{Georg Brasseu}

was born in Vienna, Austria, in 1953. He received the Dipl.-Ing. degree in electrical engineering and the doctoral degree in technical science from Vienna University of Technology, Austria, in 1979 and 1985, respectively. Since 1999, he has been a Full Professor with the Institute of Electrical Measurement and Measurement Signal Processing, Graz University of Technology (TU Graz), Austria. From 2001 to 2008, he chaired the Christian Doppler Laboratory for Automotive Measurement Research, and in 2004 and 2005, he was the Dean of the Faculty of Electrical and Information Engineering, TU Graz. Since the 1990s he has been serving for the IEEE Instrumentation and Measurement Society as conference, workshop, chapter and technical committee chair and co-chair, AdCom member and vice president. Since July 2013 he has been President of the Division of Mathematics and the Natural Sciences at the Austrian Academy of Sciences. He has authored or co-authored over 100 technical papers, holds 48 patents and has received numerous scientific research awards. His research interests focus on automotive electronics, sensors and actuators, capacitive-sensing devices, analog circuit design and sustainable mobility. 